\title{
Identification of a Meckel's Diverticulum by Wireless Capsule Endoscopy
}

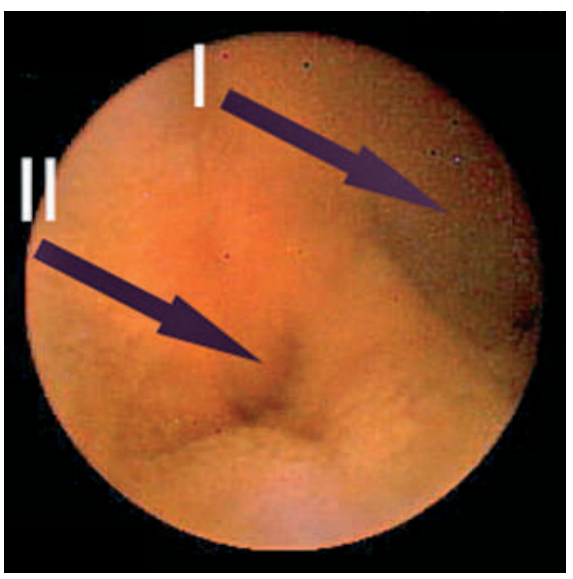

Figure 1 A 13-year-old patient was admitted to our hospital due to recurrent severe gastrointestinal bleeding. Prior to admission a further severe bleeding occured, causing a minimum hemoglobin level of $6.0 \mathrm{~g} / \mathrm{dl}$. Upper gastrointestinal endoscopy, colonoscopy, magnetic resonance enteroclysis, and Meckel's scan had not identify the bleeding site. Wireless capsule endoscopy (WCE; Given M2A, Given Imaging, Yoqneam, Israel) was performed as the next diagnostic procedure. WCE did not demonstrate an active bleeding, but identified a small diverticular like orifice in the middle part of the ileum (arrow II). The capsule video endoscope rested for a few seconds above this orifice. Small bowel lumen (arrow I) was identified following further propulsion of the endoscope.

\section{S. Gölder, J. Schmidt, P. Kolmsee,}

\section{K. Rösner, M. Strik, W. Mohren,}

N. Weigert

${ }^{1}$ Department of Internal Medicine I, Klinikum St. Elisabeth, Straubing, Germany

${ }^{2}$ Department of Surgery I, Klinikum St. Elisabeth, Straubing, Germany

${ }^{3}$ Department of Pathology, Klinikum St. Elisabeth, Straubing, Germany

Video 1 Identification of a Meckel's Diverticulum by Wireless Capsule Endoscopy.

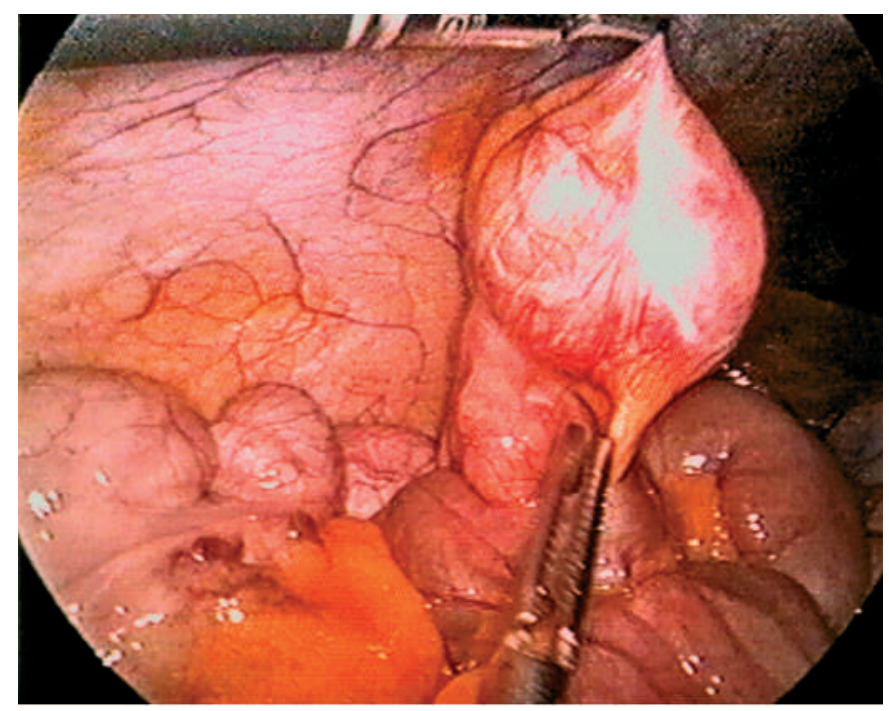

Figure 2 Laparoscopy was therefore performed, and demonstrated a Meckel's diverticulum $100 \mathrm{~cm}$ from the ileocecal valve.
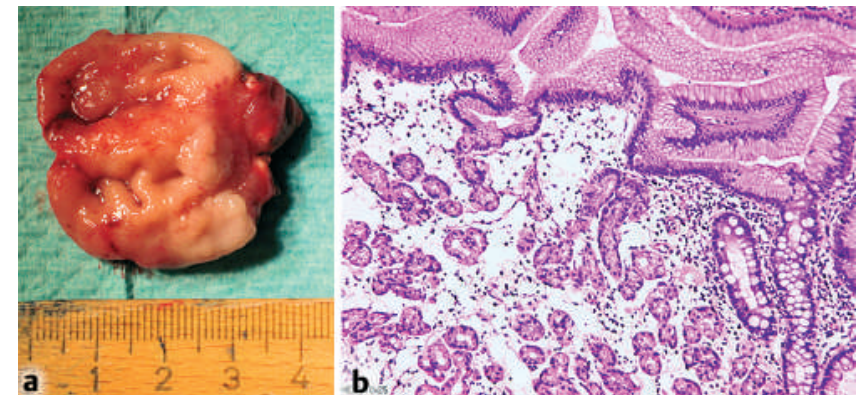

Figure 3 a A Meckel's diverticulum of length $4 \mathrm{~cm}$ was resected by minilaparotomy. b Histological examination showed ectopic gastric mucosa inside the diverticulum (hematoxylin and eosin, 1:250). After resection of the Meckel's diverticulum no further gastrointestinal bleeding occurred. In our patient, WCE demonstrated a Meckel's diverticulum with ectopic gastric mucosa, without active bleeding, despite negative findings from a Meckel scan.

\section{Corresponding Author}

\section{S. Gölder, M.D.}

Department of Internal Medicine I

Klinikum St. Elisabeth

D-94315 Straubing

Germany

Fax: $\quad+49-9421-7101574$

E-mail: goelders@klinikumstraubing.de 